\title{
Combined DPD and UGT1A1 Mutation in a Single Patient: A Case Report
}

\section{Lalkota Prakash Bhanu*, Srinivasa BJ', Mohammad Nasiruddin² and Naik Radheshyam ${ }^{1}$}

${ }^{1}$ Department of Medical Oncology, HCG Cancer Speciality Centre, Bangalore, Karnataka, India

${ }^{2}$ Department of Molecular Pathology, HCG Cancer Speciality Centre, Bangalore, Karnataka, India

\begin{abstract}
Pharmacogenetic studies on 5-FU have mostly focused on enzyme Dihydropyrimidine Dehydrogenase (DPD), which is a rate-limiting enzyme in uracil and thymine catabolic pathway. Its activity has been found to be highly variable in different populations and several DPYD polymorphisms have been reported to be responsible for the decrease in activity of enzyme function and a high risk of 5-FU toxicity. Studies on 5-FU have mostly focused on t UGT1A1 genetic variations have been extensively investigated in relation to hyperbilirubinemia syndromes, as the UGT1A1 enzyme catalyses bilirubin glucuronidation. The importance of glucuronidation pathway in irinotecan treatment, UGT1A1 was chosen as the candidate gene to be investigated as a predictor of severe toxicity. Existence of combined mutation of DPD and UGT1A1 is adversely increases the treatment toxicity. A 57 year old male patient was diagnosed moderately differentiated adenocarcinoma rectum and patient was evaluated with DPD gene mutation and found positive for Heterozygous (496A19), Homozygous (855/C) and Heterozygous (1627). Thereafter, the treatment regimen was changed to IROX, patient's conditions worsen progressively with Grade IV neutropenia and further complicated by sepsis. Patient was evaluated for UGT1A1 gene mutation. Subsequently, UGT1A1*1 and UGT1A1*28 gene was found to be mutated (Heterozygous).
\end{abstract}

Keywords: DPD; UGT1A1; 5FU; Irinotecan; Pharmacogenetics

\section{Introduction}

Fluorouracil (5-FU)-based therapy is employed in various oncology treatments such as GI, breast and aero-digestive tract cancer.

Fluorouracil (5-FU) therapy works by inhibition of thymidylate synthase and the metabolic impairment of DNA/RNA through its metabolite incorporation. The mechanism of action is influenced by the different modes of administration of 5-FU (infusion, bolus) [1,2].

Depending on the regimen of 5-FU therapy used some (10-30\%) of patients also reported grade 3-4 toxicities with reported adverse effects such as myelosuppression, diarrhoea, mucositis and hand-foot syndrome [3].

A pharmacogenetics study of 5-FU metabolism depends on the rate-limiting catabolic enzyme, Dihydropyrimidine Dehydrogenase (DPD). DPD catabolizes some $85 \%$ of administered 5-FU with its impairments causes accumulation of 5-FU anabolites, thus causing toxicities in treated patients [4]. In general population frequency of DPD mutation is $1-2 \%$ [5].

Most of them adverse reactions of fluoropyrimidines are due to inter-individual genetic variations of DPD. Therefore its role in 5-FU therapies needs to be largely investigated. DPD activity is in fact highly variable in the population and its decreases enzyme activity and increased risk of 5-FU toxicity have been shown to be associated with several DPYD polymorphism. It is reported that 3-5\% individual have been detected with DPD activities [6].

Irinotecan the semisynthetic derivative of camptothecin works by inhibition of ultracellular topoisomerase-1. This drug is metabolized by carboxylation into its active metabolite $\mathrm{SN}-38$, which is in turn inactivated by uridine diphosphate glucorosyl transferase 1 (UGT1A1) into SN-38G and is excreted in the bile [7]. The most common irinotecan side effects include neutropenia, nausea, alopecia, febrile neutropenia and delayed diarrhoea. This main and dose limiting toxicity is due to $\beta$-glucuronidase in the bowel which reactivates $\mathrm{SN}-384$ into its active metabolite SN-38 $[8,9]$.
UGT1A1 genetic variations have been extensively investigated in relation to hyperbilirubinemia syndromes, as the UGT1A1 enzyme catalyses bilirubin glucuronidation.

There is a polymorphism in the promoter of UGT1A1 gene where seven instead of 6 TA repeats are found in the promoter region which causes most cases of this disorder. This polymorphism has been given the name $U G T 1 A 1^{\star} 28$ [10]. The importance of glucuronidation pathway in irinotecan treatment, UGT1A1 was chosen as the candidate gene to be investigated as a predictor of severe toxicity.

In the present study we report a case study of a patient suffering with adenocarcinoma of rectum where there is a combined pharmacogenetics syndrome that is homo with heterozygous for DPD gene $(496 \mathrm{~A} / \mathrm{G})$, $(855 / \mathrm{C})$ in the presence of the heterozygous gene UGT1A1.

\section{Case Report}

A 57 year old male patient from India was diagnosed moderately differentiated adenocarcinoma rectum with grade II in 2015. Patient underwent abdominal perianal resection followed by concurrent chemo radiation. The patient's treatment was started on Folfox-IV (Oxaliplatin, Leucovorin, 5FU) regimen, after 5-FU administration patient got Grade II diarrhoea \& Grade II neutropenia after cycle 1 and Grade II diarrhoea with Grade III neutropenia after cycle 2 (CTCAE V4.0). Even after reducing dose of 5-FU, patient got diarrhoea and neutropenia. Patient was evaluated with DPD gene mutation and found positive

*Corresponding author: Lalkota Prakash Bhanu, Department of Medical Oncology, HCG Cancer Speciality Centre, Bangalore, Karnataka, India, Tel: 09739699388; E-mail: bhanu.lalkota@gmail.com

Received December 14, 2016; Accepted February 06, 2017; Published February 14, 2017

Citation: Bhanu LP, Srinivasa BJ, Nasiruddin M, Radheshyam N (2017) Combined DPD and UGT1A1 Mutation in a Single Patient: A Case Report. JPharmacogenomics Pharmacoproteomics 8: 165. doi: 10.4172/2153-0645.1000165

Copyright: ( 2017 Bhanu LP, et al. This is an open-access article distributed under the terms of the Creative Commons Attribution License, which permits unrestricted use, distribution, and reproduction in any medium, provided the original author and source are credited. 
for Heterozygous (496A19), Homozygous (855/C) and Heterozygous (1627) (Table 1). Thereafter, the treatment regimen was changed to IROX (Irinotecan and Oxaliplatin) regimen, after starting IROX regimen patient got Grade II diarrhoea and Grade II Neutropenia in 2 cycles. After dose reduction in next cycle, patient's conditions worsen progressively with Grade IV neutropenia and further complicated by sepsis. After better ICU medical care patient came out from the sepsis. Keeping an account of IROX toxicity in mind, further patient was evaluated for $U G T 1 A 1$ gene mutation. Subsequently, $U G T 1 A 1^{\star} 1$ and $U G T 1 A 1^{\star} 28$ gene was found to be mutated (Heterozygous) (Table 2).

\section{Discussion}

$5 \mathrm{FU}$ is often the choice drug for colorectal cancer treatments. DPD activity is one of the main factors in determining drug exposure since $80 \%$ of the dose is metabolized by this enzyme in $5 \mathrm{FU}$ degradation [11]. PBMCs are often used as a surrogate for liver DPD activity as they are easily accessible [11] even though it is the liver which is primarily responsible for DPD activity for majority of 5-FU catabolism. The structural organization of DPD gene entails a $150 \mathrm{~kb}$ length with 23 exons ranging in size from 69-1404 bp [12]. Mutation in DPYD gene can be easily detected by the advent of rapid assays prior to the start of chemotherapy with 5FU. It is however important to identify those mutation that result in defective DPD protein. 19 molecular defects in the DPYD gene have been reported of late such as point/deletion due to exon skipping have been reported, but not all mutations results in DPD enzyme activity [12]. Lethal outcomes have also been reported in several heterozygous $D P Y D^{\star} 2 A$ mutation carries due to severe $5 \mathrm{FU}$ induced toxicity. In addition fatal 5FU induced toxicities have also been reported in patients with complete DPS deficiency having innate homozygous $D P Y D^{\star} 2 A$ mutations [5]. In an effort to identify relevant inherited variations to better predict patient response to chemotherapy new pharmacogenomics approaches have been initiated.

Genetic variations include nucleotide repeats, insertions, deletions, and Single Nucleotide Polymorphisms (SNPs), which can alter the amino acid sequence of the encoded proteins, RNA splicing, and gene transcription [13].

Functional changes in phenotype like changes in expression levels and/or activity of expressed proteins and their role in drug response has been extensively investigated in genetic polymorphisms of drugmetabolizing enzymes, transporters and molecular targets. The Uridine Diphosphate Glucuronosyltransferase (UDP-Glucuronosyltransferase or UGT) belong to a super family of enzymes which perform a reaction called as glucuronidation where it conjugates glucuronic acid and can metabolize bilirubin, steroids and certain drugs like irinotecan. Thus, genetic variation in UGT1A1 correlates with adverse events caused by irinotecan toxicity [14].

\begin{tabular}{|c|c|c|}
\hline Genotype & Effect of Polymorphism & DPD Genotype status \\
\hline 85 T/C DETECTED & No enzyme activity & Homozygous \\
\hline 496 A/G: DETECTED & Reduced enzyme activity & Heterozygous \\
\hline 1627: DETECTED & Reduced enzyme activity & Heterozygous \\
\hline
\end{tabular}

Table 1: Dihydropyrimidine (DPD) gene mutation (Sequencing).

\begin{tabular}{|c|c|c|}
\hline Genotype & Effect of polymorphism & $\begin{array}{c}\text { UGT1A1 Genotype } \\
\text { status }\end{array}$ \\
\hline UGT1A1*1 A(TA)6TAA & Normal enzyme activity & Heterozygous Detected \\
\hline UGT1A1*28 A(TA)7TAA & Minimal enzyme activity & Heterozygous Detected \\
\hline UGT1A1*6 G71R & Reduced enzyme activity & Not Detected \\
\hline UGT1A1*7Y486D & Reduced enzyme activity & Not detected \\
\hline
\end{tabular}

Table 2: UGT1A1 genotyping for irinotecan metabolism (PCR and sequencing).
There are over 113 reported genetic variations of UGTIA1. $U G T I A 1^{*} 1$ is denoted as the wild type allele and is linked to normal enzyme activity. Whereas, $U G T 1 A 1^{\star} 28$ is the most common variant allele and is found most frequently in African-Americans (0.42-0.45 allele frequency) and Caucasians (0.26-0.31), the most, and is less frequent in Asian populations (0.09-0.16) and these cases present a higher probability for irinotecan toxicity in blood $[10,14,15]$.

In 2005, US FDA recommended that patients who are homozygous for $U G T 1 A 1^{\star} 28$ will have high risk by Irinotecan toxicity, and warn that patient should receive reduced starting doses.

Severe toxicity in a previous index patient who was DPD deficient could be attributed to the mutation in UGTIA1 promotor. Coexistence of both DPD and UGT1A1 mutation was reported in a single patient [11] till now as per literature search. This is one more rare case reporting of combined genetic syndrome and also for both mutation patient had heterozygous and homozygous association.

It is therefore essential that we advance our knowledge to first predict and then prevent the occurrence of toxicities related to $5 \mathrm{FU}$. However, there is no consensus on screening of DPYD mutations prior to 5-FU treatment. Many DPYD allele variants have been discovered, but there has been a lack of consistency between the genetic variant and the degree of toxicity; many such individuals have toxicity profiles similar to those of the general population.

Whenever the patient has unexplained Neutropenia and Grade III \& IV Gastrointestinal toxicity with standard dose of FOLFOX/ IRINOTECAN, we should consider investigating the UGT1A1 mutation analysis.

\section{Conclusion}

Existence of combined mutation of DPD and UGT1A1 is adversely increases the treatment toxicity. Though simultaneous testing for the same is not recommended, one should consider the possibility of whether patient is intolerant to commonly used 5FU and Irinotecan based chemotherapy or not. Treatment efficacy of this combined pharmacogenetics syndrome is uncertain.

\section{References}

1. Diaz-Rubio E, Tabernero J, Gomez-Espana A, Massutí B, Sastre J, et al. (2007) Phase III study of capecitabine plus oxaliplatin compared with continuousinfusion fluorouracil plus oxaliplatin as first-line therapy in metastatic colorectal cancer: final report of the Spanish Cooperative Group for the Treatment of Digestive Tumors Trial. J Clin Oncol 25: 4224-4230.

2. Spears CP, Gustavsson BG, Mitchell MS, Spicer D, Berne M (1984) Thymidylate synthetase inhibition in malignant tumors and normal liver of patients given intravenous 5-fluorouracil. Cancer Res 44: 4144-4150.

3. Falvella FS, Caporale M, Cheli S, Martinetti A, Berenato R, et al. (2015) Undetected toxicity risk in pharmacogenetic testing for dihydropyrimidine dehydrogenase. Int J Mol Sci 16: 8884-8895.

4. Diasio RB, Harris BE (1989) Clinical pharmacology of 5-fluorouracil. Clin Pharmacokinet 16: 215- 237.

5. Steiner M, Seule M, Steiner B, Bauer I, Freund M, et al. (2005) 5-Fluorouracil/ irinotecan induced lethal toxicity as a result of a combined pharmacogenetic syndrome: report of a case. J Clin Pathol 58: 553-555.

6. Etienne MC, Lagrange JL, Dassonville O (1994) Population study of dihydropyrimidine dehydrogenase in cancer-patients. J Clin Oncol 12: 22482253.

7. Folprecht G, Kohne $\mathrm{CH}$ (2004) The role of new agents in the treatment of colorectal cancer. Oncology 66: 1-17.

8. Vanhoefer U, Harstrick A, Achterrath W, Cao S, Seeber S, et al. (2001) Irinotecan in the treatment of colorectal cancer: clinical overview. J Clin Oncol 19: 1501-1518. 
Citation: Bhanu LP, Srinivasa BJ, Nasiruddin M, Radheshyam N (2017) Combined DPD and UGT1A1 Mutation in a Single Patient: A Case Report. J Pharmacogenomics Pharmacoproteomics 8: 165. doi: 10.4172/2153-0645.1000165

Page 3 of 3

9. Kawato $Y$, Aonuma M, Hirota $Y$, Kuga H, Sato $K$ (1991) Intracellular roles of $\mathrm{SN}-38$, a metabolite of the camptothecin derivative CPT-11, in the antitumor effect of CPT-11. Cancer Res 51: 4187-4191.

10. Beutler E, Gelbart T, Demina A (1998) Racial variability in the UDPglucuronosyltransferase 1 (UGT1A1) promoter: a balanced polymorphism for regulation of bilirubin metabolism? P Natl Acad Sci U S A 95: 8170- 8174

11. Diasio RB, Lu Z (1994) Dihydropyrimidine dehydrogenase activity and fluorouracil chemotherapy. J Clin Oncol 12: 2239-2242.

12. Maring JG, van Kuilenburg ABP, Haasjes J, Piersma H, Groen HJM, et al. (2002) Reduced 5-FU clearance in a patient with low DPD activity due to heterozygosity for a mutant allele of the DPYD gene. Br J Cancer 6: 1028-1033.

13. Lee W, Lockhart AC, Kim RB, Rothenberg ML (2005) Cancer Pharmacogenomics: Powerful Tools in Cancer Chemotherapy and Drug Development. Oncologist 10: 104-111.

14. Innocenti F, Undevia SD, Iyer L, Chen PX, Das S, et al. (2004) Genetic variants in the UDP-glucuronosyltransferase $1 \mathrm{~A} 1$ gene predict the risk of severe neutropenia of irinotecan. J Clin Oncol 22: 1382-1388.

15. Strassburg CP (2008) Pharmacogenetics of Gilbert's syndrome Pharmacogenomics 9: 703-715. 